\title{
Transient Mixed Convection Boundary Layer Flow of an Incompressible Fluid Past a Wedge in Presence of Magnetic Field
}

\author{
Shayma Joya Saha, Litan Kumar Saha* \\ Department of Applied Mathematics, University of Dhaka, Dhaka, Bangladesh \\ Email address: \\ lksaha.math@gmail.com (L. K. Saha) \\ ${ }^{*}$ Corresponding author
}

To cite this article:

Shayma Joya Saha, Litan Kumar Saha. Transient Mixed Convection Boundary Layer Flow of an Incompressible Fluid Past a Wedge in Presence of Magnetic Field. Applied and Computational Mathematics. Vol. 8, No. 1, 2019, pp. 9-20. doi: 10.11648/j.acm.20190801.13

Received: February 1, 2019; Accepted: March 11, 2019; Published: March 25, 2019

\begin{abstract}
In this paper, an analysis is performed to explorethe transient, laminar two-dimensional, mixed convection boundary layer flow of a viscous and incompressible fluid past a vertical wedge taking into account the effect of magnetic field. With appropriate transformations the boundary layer equations are reduced to a local nonsimilarity equations and the solutions are obtained employing three distinct methods, namely, (i) perturbation method for small time; (ii) asymptotic solution method for large time; (iii) straight forward finite difference method for any time. The agreement between the solutions obtained from prescribed methods is found to be excellent. In this study the evaluation of skin-friction coefficient and the local Nusselt number with the effects of different governing parameters such as different time, $\tau$, the exponent, $m(=0.4,0.5,1.0)$, mixed convection parameter, $\lambda(=0.0,0.2,0.4)$ and magnetic field parameter, $M(=0.0,1.0)$ for fluids having Prandtl number, $P r=0.72,1.0$ and 7.0have been discussed. It is observed that both the local skin friction and local Nusseltnumber decreases due to an increase in the value of $M$. It is also found that an increase in the value of Prandtl number, $\operatorname{Pr}$, leads to a decrease in the value of local skin friction coefficient and the value of local Nusselt number coefficient increases with the increasing values of Prandtl number.
\end{abstract}

Keywords: Transient Flow, Mixed Convection, Magnetohydrodynamics, Boundary Layer, Wedge Flow

\section{Introduction}

The laminar boundary layer flow of an incompressible fluid past bodies of different geometries has been studied with a great importance because it has a considerable curiosity among scientists and researchers. Many practical applications can be provided by incompressible boundary layer flow over two dimensional or axisymmetric bodies. The steady Falkner-Skan problem has beenstudied by many investigators such as Leal [1], Gersten and Herwig [2], Schlichting and Gersten [3]. The skin friction and heat transfer in two-dimensional, viscous, incompressible laminar flow over wedge-shaped bodies can be calculated accurately by solving the boundary-layer equations. Falkner and Skan [4] first deduced the momentum boundary layer equation for Falkner-Skan flow past a wedge, with potential flow velocity $u_{e}(x)=U_{0} x^{m}$. Later, Hartree [5] investigated the similarity solutions of the flow in detail. He obtained the solutions in terms of velocity distribution for different values of pressure gradient parameter. For flow over an arbitrary body shape with known pressure or velocity distribution where there exists no similarity, the skin friction and heat transfer are conventionally found by an approximate method, either the integral method or the equivalent wedge flow approximation. For most engineering applications, sufficiently accurate result can be obtained using both of these two methods. It is necessary to have the solutions of the boundary-layer equations for wedge type flows to apply the equivalent wedge flow method for the prediction of skin-friction and heat transfer. The skin friction and heat transfer for incompressible laminar flow over porous wedges with suction subjected to variable wall temperaturehave been obtained Koh and Hartnett [6]. The similarity solution had been obtained when the variations of the wall temperature and the suction rate are proportional to power-law of $x$. From the practical point of view, however, the surface mass-flux with constant velocity may be more easily realized than with $x^{(m-1) / 2}$, where $x$ is the distance from the leading edge, $m$ the pressure gradient parameter.

The transient conditions of motion in fluids have become 
very important in recent years due to its application in some engineering fields of aerodynamics and hydrodynamics. There is a huge volume of literature on unsteady, forced convection boundary-layer flows past bodies of different geometries that give rise to the Falkner-Skan equations, see, for example, Riley [11], Telionis [12], and Ludlow et al. [13]. When the fluid is assumed to have constant properties, the problem reduces to an uncoupled, laminar boundary-layer flow and any changes in the temperature do not affect the fluid velocity field. When the thermo physical fluid properties depend on the temperature the problem becomes coupled and then the fluid velocity is also a function of time. Smith [14] initiated the study of the unsteady, incompressible forced convection, boundary-layer flow past a semi-infinite wedge impulsively set into motion. This problem was subsequently solved numerically by Nanbu [15] using the method proposed by Hall [17] and later it was modified by Harris et al. [18]. Laminar boundary-layer flow over wedge with suction/injection has been discussed by Kafoussias and Nanousis [7] and Devi and Kandasamy [29]. Hossain et al. [9] examined a steady two dimensional laminar forced flow of a viscous incompressible fluid past a horizontal wedge with uniform surface heat flux.

The combined effect of both free and forced convection, which is known as mixed convection has also been the focus of research because of its technical applications. Kumari and Gorla [8] carried out a boundary-layer analysis considering the combined convection along a vertical non-isothermal wedge embedded in a fluid-saturated porous medium. A steady mixed convection boundary layer flow over a vertical wedge with the effect of magnetic field embedded in a porous medium has been studied by Kumari et al. [10]. The unsteady mixed convection boundary-layer flow along a symmetric wedge with variable surface temperature has been analyzed by Hossain et al. [16]. In their analysis, Hossain et al. [16] obtained the solution of the problem in terms of skin friction and heat transfer using the implicit finite difference method together with the Keller box elimination technique [19].

A combination of fluid mechanics and electro-magnetism is regarded as magnetohydrodynamics (MHD). It is the behavior of electrically conducting fluid in the presence of magnetic field. The study of the flow of electrically conducting fluid in the presence of magnetic field is important from a technical point of view. Due to the growing demand of technological appliances, there are many cases in which magnetic fields are strongly encountered in an electrically conducting fluid, for example, electric power generation, astrophysical flows, solar power technology, space vehicle re-entry, nuclear engineering applications, etc. The MHD boundary-layer flow, which finds its application in nuclear reactors and in the boundary layer control in the field of aeronautics is extensively studied by Sparrow and Cess [20], Singh and Cowling [21] and Wilks [22]. Wilks [22] provides the ground on which solution for large $x$ can be obtained via matched asymptotic expansion method with the aim of achieving consistency. Cobble [23] analyzed the solution using the similarity analysis considering the effects of a magnetic field. Later, Hossainet al. [24] analyzed the combined forced and free convection boundary-layer flow of an electrically conducting fluid in the presence of transverse magnetic field. Ganesan and Palani [25] and Palani and Kim [26] proposed a study of the problem of unsteady natural convection flow of a viscous incompressible electrically conducting fluid past an inclined plate, under the influence of magnetic field.

Motivated by the aforementioned investigations, the present analysis is devoted to study the transient, laminar mixed convection boundary-layer flow of an incompressible, viscous fluid past a wedge with the presence of magnetic field. The governing boundary layer equations are reduced to non-linear partial differential forms usingappropriate transformations. The transformed boundary layer equations are solved numerically using three distinct methods, namely the finite difference method for all time regime, the perturbation technique for small time regime and the asymptotic solutions for large time regime. All the solutions are obtained in terms of skin friction and Nusselt number for different values of Prandtl number $P r$, magnetic parameter $M$, pressure gradient parameter $m$ and the Richardson number $\lambda$, which is the ratio between $G r_{L}$ and $R e_{L}^{2}$. The effects of varying $M, m$, Prand $\lambda$, on the shearing stress and the heat transfer rate in terms of skin friction and Nusselt number, respectively, are shown both in tabular and graphical forms.

\section{Mathematical Formulation}

A two-dimensional, unsteady, laminar mixed convection flow of a viscous incompressible electrically conducting fluid through a uniformly distributed transverse magnetic field of strength $B_{0}$ past a wedge with a distributed heat source is considered. Due to buoyancy effects the flow over the wedge develops instantaneously and its velocity is given by,

$$
\bar{u}_{e}(\bar{x})=U_{\infty}\left(\frac{\bar{x}}{L}\right)^{m} \text { for } m \leq 1
$$

The flow configuration and coordinate system are shown in Figure 1.

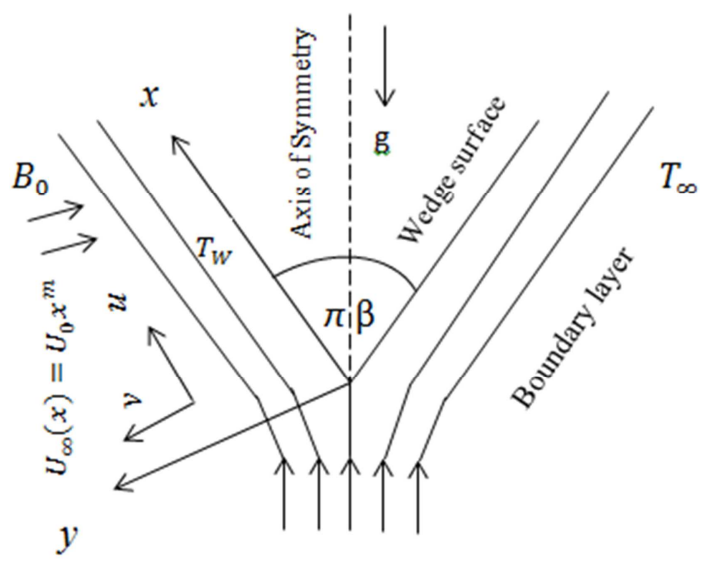

Figure 1. Flow model with coordinate system.

The Lorentz force, $F$, is a result of the magnetic field effects 
on the electrically conducting fluid and is determined from the following equations:

$$
\frac{\partial \bar{u}}{\partial \bar{x}}+\frac{\partial \bar{v}}{\partial \bar{y}}=0
$$

$$
\begin{gathered}
\frac{\partial \bar{u}}{\partial \bar{t}}+\bar{u} \frac{\partial \bar{u}}{\partial \bar{x}}+\bar{v} \frac{\partial \bar{u}}{\partial \bar{y}}=\bar{u}_{e} \frac{\partial \bar{u} e}{\partial \bar{x}}+v \frac{\partial^{2} \bar{u}}{\partial \bar{y}^{2}}+g \beta\left(\bar{T}-T_{\infty}\right)-\frac{\sigma B_{0}^{2}}{\rho}\left(\bar{u}-\bar{u}_{e}\right) \\
\frac{\partial \bar{T}}{\partial \bar{t}}+\bar{u} \frac{\partial \bar{T}}{\partial \bar{x}}+\bar{v} \frac{\partial \bar{T}}{\partial \bar{y}}=\frac{k}{\rho C_{p}} \frac{\partial^{2} \bar{T}}{\partial \bar{y}^{2}} \quad \text { (3) } \quad x=\frac{\bar{x}}{L}, y=\operatorname{Re}_{L}{ }^{1 / 2} \frac{\bar{y}}{L}, u=\frac{\bar{u}}{U_{\infty}}, v=\operatorname{Re}_{L}^{1 / 2} \frac{\bar{v}}{U_{\infty}} \\
\text { Here } \bar{x} \text { and } \bar{y} \text { are the coordinates along with and } \quad u_{e}=\frac{\bar{u}_{e}}{U_{\infty}}, t=\frac{U_{\infty}}{L} \bar{t}, T=\frac{T_{W}-T_{\infty}}{T_{r}-T_{\infty}}, R_{L}=\frac{U_{\infty} L}{v}
\end{gathered}
$$
perpendicular to the wedge surface, $\bar{u}$ and $\bar{v}$ are the velocity components associated with the direction of increasing coordinates $\bar{x}$ and $\bar{y}$ respectively, $\bar{t}$ is the time variable, $\bar{T}$ is the fluid temperature in the boundary layer, $T_{W}$ and $T_{\infty}$ are the temperature of the wall and ambient fluid respectively, $\rho$ is the density, $\sigma$ is the electrical conductivity, $k$ is the thermal conductivity of the fluid, $\mu$ is the coefficient of viscosity, $g$ is the acceleration due to gravity, $\beta$ is the coefficient of volumetric expansion, $C_{p}$ is the specific heat at constant pressure, $L$ is the characteristic length and $m$ is the pressure gradient related to the included angle $\pi \beta$ by $m=\beta /(2-$ $\beta)$.

The boundary conditions for the present problem are:

$$
\begin{gathered}
\bar{u}=0, \bar{v}=0, \bar{T}=T_{w}(\bar{x}) \text { at } \bar{y}=0 \\
\bar{u}=\bar{u}_{e}(\bar{x}), \bar{T} \rightarrow 0 \text { as } \bar{y} \rightarrow \infty
\end{gathered}
$$

The velocity over the wedge is now given by

$$
u_{e}(x)=x^{m}, \text { for } m \leq 1
$$

and, on introducing the above dimensionless dependent and independent variables given in (5) in equations (1)-(3), the following dimensionless form of the governing equations is obtained:

$$
\frac{\partial u}{\partial x}+\frac{\partial v}{\partial y}=0
$$

$$
\begin{gathered}
\frac{\partial u}{\partial t}+u \frac{\partial u}{\partial x}+v \frac{\partial u}{\partial y}=u_{e} \frac{\partial u_{e}}{\partial x}+\frac{\partial^{2} u}{\partial y^{2}}+\lambda T-M^{2}\left(u_{e}-u\right) \\
\frac{\partial T}{\partial t}+u \frac{\partial T}{\partial x}+v \frac{\partial T}{\partial y}=\frac{1}{\operatorname{Pr}} \frac{\partial^{2} T}{\partial y^{2}}
\end{gathered}
$$

The boundary conditions (4) then take the form:

$$
u=0, v=0, T=x^{2 m-1} \text { at } y=0 u=u_{e}(x)=x^{m}, T \rightarrow 0 \text { as } y \rightarrow \infty \text { For } t \geq 0 \text { and } m \leq 1
$$

In the above equations, Pris the Prandtl number, $G r_{L}$ is the Grashofnumber, $R e_{L}$ is the Reynolds number, $\lambda$ is the mixed convection parameter(termed as the Richardson's number) and Mis the magnetic field parameter, which are defined as

$$
\operatorname{Pr}=\frac{\mu C_{p}}{k}, G r_{L}=\frac{g \beta\left(T_{r}-T_{\infty}\right) L^{3}}{v^{2}}, R e_{L}=\frac{U_{\infty} L}{v}, \lambda=\frac{G r_{L}}{\operatorname{Re}_{L}^{2}} \text { and } M^{2}=\frac{\sigma B_{0}^{2} L}{\rho U_{\infty}}
$$

The number of independent variables in the governing equations (6)-(8) can be reduced from three to two by introducing the non-dimensional, reduced stream function $F(\xi, \tau, \eta)$ and the non-dimensional, reduced temperature function $G(\xi, \tau, \eta)$ according to,

$$
\psi=x^{\frac{m+1}{2}}\left(2\left(1-e^{-\tau}\right)\right)^{\frac{1}{2}} F(\xi, \tau, \eta), \eta=x^{\frac{m-1}{2}}\left(2\left(1-e^{-\tau}\right)\right)^{-\frac{1}{2}} y, T=x^{2 m-1} G(\xi, \tau, \eta), \tau=x^{m-1} t \text { and } \xi=x^{1-m}
$$

Where $\eta$ is a non-dimensional similarity variable and $\psi$ is the stream function, which is defined in the usual manner $u=\frac{\partial \psi}{\partial y}$ and $v=-\frac{\partial \psi}{\partial x}$

Now, substituting the above group of transformations given in (10) into (6) to (8) one obtains the following non-similarity equations: 


$$
\begin{gathered}
F^{\prime \prime \prime}+\eta e^{-\tau} F^{\prime \prime}+\left[(m+1)\left(1-e^{-\tau}\right)+(m-1) \tau e^{-\tau}\right] F F^{\prime \prime}+2 m\left(1-e^{-\tau}\right)\left(1-F^{\prime 2}\right) \\
=2\left(1-e^{-\tau}\right)\left[\frac{\partial F^{\prime}}{\partial \tau}-\lambda G\right]+2(m-1) \tau\left(1-e^{-\tau}\right)\left[F^{\prime} \frac{\partial F^{\prime}}{\partial \tau}-F^{\prime \prime} \frac{\partial F}{\partial \tau}\right] \\
+2(1-m) \xi\left(1-e^{-\tau}\right)\left[F^{\prime} \frac{\partial F^{\prime}}{\partial \xi}-F^{\prime \prime} \frac{\partial F}{\partial \xi}\right]+2 M^{2} \xi\left(1-e^{-\tau}\right)\left(1-F^{\prime}\right) \\
\frac{1}{P r} G^{\prime \prime}+\eta e^{-\tau} G^{\prime}+\left[(m+1)\left(1-e^{-\tau}\right)+(m-1) \tau e^{-\tau}\right] F G^{\prime}-2(2 m-1)\left(1-e^{-\tau}\right) G F^{\prime} \\
=2\left(1-e^{-\tau}\right) \frac{\partial G}{\partial \tau}+2(m-1) \tau\left(1-e^{-\tau}\right)\left[F^{\prime} \frac{\partial G}{\partial \tau}-G^{\prime} \frac{\partial F}{\partial \tau}\right] \\
+2(1-m) \xi\left(1-e^{-\tau}\right)\left[F^{\prime} \frac{\partial G}{\partial \xi}-G^{\prime} \frac{\partial F}{\partial \xi}\right]
\end{gathered}
$$

The corresponding boundary conditions transform to

$$
\begin{gathered}
F(\xi, \tau, 0)=F^{\prime}(\xi, \tau, 0)=0, G(\xi, \tau, 0)=1 \\
F^{\prime}(\xi, \tau, \infty)=1, G(\xi, \tau, \infty)=0
\end{gathered}
$$

In the above equations, prime denotes differentiation of the functions with respect to $\eta$ only.

The above equation is applicable for $0 \leq \tau \leq \infty$.

In practical applications, two physical quantities of primary interest are to be determined, such as, surface shear stress and the rate of heat transfer at the surface. These may be obtained in terms of the skin friction coefficient,

$$
C_{f}=\tau_{w}(\bar{x}) / \rho\left(\bar{u}_{e}(\bar{x})\right)^{2}
$$

And the local Nusselt number,

$$
N u=q_{w}(\bar{x}) \bar{x} / k_{f}\left(T_{w}-T_{\infty}\right)
$$

Where, $\tau_{w}(\bar{x})=\mu\left(\frac{\partial \bar{u}}{\partial \bar{y}}\right)_{\bar{y}=0}$ is the shear-stress along the surface, and $q_{w}(\bar{x})=-k_{f}\left(\frac{\partial \bar{T}}{\partial \bar{y}}\right)_{\bar{y}=0}$ is the surface heat flux, where, $k$ is the thermal conductivity of the fluid, and $\mu$ is the coefficient of viscosity.

By introducing the non-dimensional variables (5) and the transformation (10), the skin friction coefficient, $C_{f} \operatorname{Re}_{x}^{\frac{1}{2}}$ and the local Nusselt number, $N u R e_{x}^{-\frac{1}{2}}$, can now be defined by

$$
\begin{gathered}
C_{f} \operatorname{Re}_{x}^{\frac{1}{2}}=\xi^{\frac{m+1}{2(m-1)}}\left(2\left(1-e^{-\tau}\right)\right)^{-\frac{1}{2}} F^{\prime \prime}(\xi, \tau, 0) \\
\operatorname{NuRe}_{x}^{-\frac{1}{2}}=-\xi^{\frac{3 m+1}{2(1-m)}}\left(2\left(1-e^{-\tau}\right)\right)^{-\frac{1}{2}} G^{\prime}(\xi, \tau, 0)
\end{gathered}
$$

After getting the values of $F^{\prime \prime}(\xi, \tau, 0)$ and $G^{\prime}(\xi, \tau, 0)$ from the solutions of the governing equations (11)-(13) we obtain the values of the skin friction coefficient and the local Nusselt number from the relations (14) and (15).

\section{Methods of Solution}

The present problem described by the dimensionless equations (11)-(13) has been solved using the perturbation method for small time regime, the asymptotic method for large time regime and finally the finite difference method for any time regime. The methods of solution adopted by Hossain et al. [16] and Mahfooz et al. [27] are employed here. In the following subsections details of the methods of solution are illustrated.

\subsection{Perturbation Solutions for Small Time Regime}

Physically, at the initial stage of transient process, the development of the momentum and thermal boundary layers are due to the dominant diffusion on convection. For this regime, the equations (11) and (12) which is valid for any time regime, reduces to the following form

$$
\begin{gathered}
F^{\prime \prime \prime}+\eta F^{\prime \prime}+2 m \tau F F^{\prime \prime}+2 m \tau\left(1-F^{\prime 2}\right)=2(m-1) \tau^{2}\left[F^{\prime} \frac{\partial F^{\prime}}{\partial \tau}-F^{\prime \prime} \frac{\partial F}{\partial \tau}\right]+2 \tau\left[\frac{\partial F^{\prime}}{\partial \tau}-\lambda G\right] \\
+2(1-m) \tau \xi\left[F^{\prime} \frac{\partial F^{\prime}}{\partial \xi}-F^{\prime \prime} \frac{\partial F}{\partial \xi}\right]+2 M^{2} \tau \xi\left(1-F^{\prime}\right) \\
\frac{1}{P r} G^{\prime \prime}+\eta G^{\prime}+2 m \tau F G^{\prime}-2(2 m-1) \tau G F^{\prime}=2(m-1) \tau^{2}\left[F^{\prime} \frac{\partial G}{\partial \tau}-G^{\prime} \frac{\partial F}{\partial \tau}\right]+2 \tau \frac{\partial G}{\partial \tau} \\
+2(1-m) \tau \xi\left[F^{\prime} \frac{\partial G}{\partial \xi}-G^{\prime} \frac{\partial F}{\partial \xi}\right]
\end{gathered}
$$


and the corresponding boundary conditions become

$$
\begin{gathered}
F(\xi, \tau, 0)=F^{\prime}(\xi, \tau, 0)=0, G(\xi, \tau, 0)=1 \\
F^{\prime}(\xi, \tau, \infty)=1, G(\xi, \tau, \infty)=0
\end{gathered}
$$

Since near the leading edge, $\tau$ is small, series solution of the equations (16) and (17) may be obtained by using the perturbation method treating $\tau$ as a perturbation parameter. Hence, we expand the functions $F(\xi, \tau, \eta)$ and $G(\xi, \tau, \eta)$ in powers of $\tau$ as given below:

$$
F(\xi, \tau, \eta)=\sum_{i=0}^{\infty} \tau^{i} F_{i}(\xi, \eta) \text { and } G(\xi, \tau, \eta)=\sum_{i=0}^{\infty} \tau^{i} G_{i}(\xi, \eta)
$$

Now, substituting the expressions given in (19) into equations (16) and (17) and picking up the terms up to the $\mathrm{O}\left(\tau^{2}\right)$, we get the following sets of equations with boundary conditions:

$$
\begin{gathered}
F_{0}^{\prime \prime \prime}+\eta F_{0}^{\prime \prime}=0 \\
\frac{1}{P r} G_{0}^{\prime \prime}+\eta G_{0}^{\prime}=0 \\
F_{0}(\xi, 0)=F_{0}^{\prime}(\xi, 0)=0, G_{0}(\xi, 0)=1, F_{0}^{\prime}(\xi, \infty)=1, G_{0}(\xi, \infty)=0 \\
F_{1}^{\prime \prime \prime}+\eta F_{1}^{\prime \prime}-2 F_{1}^{\prime}=-2 m\left(1-F_{0}^{\prime 2}+F_{0} F_{0}^{\prime \prime}\right)-2 \lambda G_{0}-2 M^{2} \xi\left(1-F_{0}^{\prime}\right) \\
\frac{1}{P r} G_{1}^{\prime \prime}+\eta G_{1}^{\prime}-2 G_{1}=-2 m F_{0} G_{0}^{\prime}+2(2 m-1) F_{0}^{\prime} G_{0} \\
F_{1}(\xi, 0)=F_{1}^{\prime}(\xi, 0)=0, G_{1}(\xi, 0)=0, F_{1}^{\prime}(\xi, \infty)=0, G_{1}(\xi, \infty)=0 \\
\frac{1}{P r} G_{2}^{\prime \prime \prime}+\eta F_{2}^{\prime \prime}-4 F_{2}^{\prime}=2\left[(1-2 m) F_{1} F_{0}^{\prime \prime}+(3 m-1) F_{0}^{\prime} F_{1}^{\prime}-m F_{0} F_{1}^{\prime \prime}-\lambda G_{1}\right]-2 M^{2} \xi F_{1}^{\prime}+2(1-m) \xi\left[F_{0}^{\prime} \frac{\partial F_{1}^{\prime}}{\partial \xi}-F_{0}^{\prime \prime} \frac{\partial F_{1}}{\partial \xi}\right] \\
F_{2}(\xi, 0)=F_{2}^{\prime}(\xi, 0)=0, G_{2}(\xi, 0)=0, F_{2}^{\prime}(\xi, \infty)=0, G_{2}(\xi, \infty)=0 \\
\left.\frac{\partial G_{1}}{\partial \xi}-F_{0}^{\prime \prime} \frac{\partial F_{1}}{\partial \xi}\right]
\end{gathered}
$$

In these equations, primes denote differentiation with respect to $\eta$. Solutions of the above set of equations are obtained numerically employing the sixth order implicit Runge-Kutta-Butcher [34] initial solver together with the Nachtsheim-Swigert iteration scheme [35]. Knowing the values of $F_{0}^{\prime \prime}, F_{1}^{\prime \prime}, F_{2}^{\prime \prime}, G_{0}^{\prime}, G_{1}^{\prime}$, and $G_{2}^{\prime}$ from the solutions of the equations (20)-(28), the values of the skin friction coefficient and that of the local Nusselt number can be obtained from the following expressions:

$$
\begin{aligned}
C_{f} \operatorname{Re}_{x}^{1 / 2} & =\xi^{\frac{m+1}{2(m-1)}}(2 \tau)^{-1 / 2}\left[F_{0}^{\prime \prime}(\xi, 0)+\tau F_{1}^{\prime \prime}(\xi, 0)+\tau^{2} F_{2}^{\prime \prime}(\xi, 0)\right] \\
N u e_{x}^{1 / 2} & =-\xi^{\frac{3 m+1}{2(1-m)}}(2 \tau)^{-1 / 2}\left[G_{0}^{\prime}(\xi, 0)+\tau G_{1}^{\prime}(\xi, 0)+\tau^{2} G_{2}^{\prime}(\xi, 0)\right]
\end{aligned}
$$

The values of the skin-friction, $C_{f} R e_{x}^{1 / 2}$, and the Nusselt number, $N u R e_{x}^{1 / 2}$, are obtained from the expressions(29) and (30).

\subsection{Solutions for Large Time Regime}

For large time, i.e. when $\tau>>1$, values of $e^{-\tau}=\tau^{-2}$ and $1-e^{-\tau}=1$. Thus the equations (11)-(13) take the following forms: 


$$
\begin{gathered}
F^{\prime \prime \prime}+\tau^{-2} \eta F^{\prime \prime}+\left[(m+1)+(m-1) \tau^{-1}\right] F F^{\prime \prime}+2 m\left(1-F^{\prime 2}\right)=2\left[\frac{\partial F^{\prime}}{\partial \tau}-\lambda G\right] \\
+2(m-1) \tau\left[F^{\prime} \frac{\partial F^{\prime}}{\partial \tau}-F^{\prime \prime} \frac{\partial F}{\partial \tau}\right]+2(1-m) \xi\left[F^{\prime} \frac{\partial F^{\prime}}{\partial \xi}-F^{\prime \prime} \frac{\partial F}{\partial \xi}\right]+2 M^{2} \xi\left(1-F^{\prime}\right) \\
\frac{1}{P r} G^{\prime \prime}+\tau^{-2} \eta G^{\prime}+\left[(m+1)+(m-1) \tau^{-1}\right] F G^{\prime}-2(2 m-1) G F^{\prime}=2 \frac{\partial G}{\partial \tau} \\
+2(m-1) \tau\left[F^{\prime} \frac{\partial G}{\partial \tau}-G^{\prime} \frac{\partial F}{\partial \tau}\right]+2(1-m) \xi\left[F^{\prime} \frac{\partial G}{\partial \xi}-G^{\prime} \frac{\partial F}{\partial \xi}\right]
\end{gathered}
$$

and the corresponding boundary conditions reduces to

$$
\begin{gathered}
F(\xi, \tau, 0)=F^{\prime}(\xi, \tau, 0)=0, G(\xi, \tau, 0)=1 \\
F^{\prime}(\xi, \tau, \infty)=1, G(\xi, \tau, \infty)=0
\end{gathered}
$$

Hence, we expand the functions $F(\xi, \tau, \eta)$ and $G(\xi, \tau, \eta)$ in powers of $\tau$ as given below:

$$
F(\xi, \tau, \eta)=\sum_{i=0}^{\infty} \tau^{i} F_{i}(\xi, \eta) \text { and } G(\xi, \tau, \eta)=\sum_{i=0}^{\infty} \tau^{i} G_{i}(\xi, \eta)
$$

Now, substituting the expressions given in (34) into equations (31) and (32) and equating the terms of like powers of $\tau$ to zero, we get the following sets of equations:

$$
\begin{gathered}
F_{0}^{\prime \prime \prime}+(m+1) F_{0} F_{0}^{\prime \prime}+2 m\left(1-F_{0}^{\prime 2}\right)=-2 \lambda G_{0}+2(1-m) \xi\left[F_{0}^{\prime} \frac{\partial F_{0}^{\prime}}{\partial \xi}-F_{0}^{\prime \prime} \frac{\partial F_{0}}{\partial \xi}\right]+2 M^{2} \xi\left(1-F_{0}^{\prime}\right) \\
\frac{1}{P r} G_{0}^{\prime \prime}+(m+1) F_{0} G_{0}^{\prime}-2(2 m-1) G_{0} F_{0}^{\prime}=2(1-m) \xi\left[F_{0}^{\prime} \frac{\partial G_{0}}{\partial \xi}-G_{0}^{\prime} \frac{\partial F_{0}}{\partial \xi}\right] \\
F_{0}(\xi, 0)=F_{0}^{\prime}(\xi, 0)=0, G_{0}(\xi, 0)=1, F_{0}^{\prime}(\xi, \infty)=1, G_{0}(\xi, \infty)=0
\end{gathered}
$$

In these equations, primes denote differentiation with respect to $\eta$. Since the equation (35)-(37) are non-similar equations; in order to solve these non-similar equations we have adapted the local nonsimilarity method described in the former chapter. At the second order of truncation, the terms involving derivatives of $\xi$ have been neglected. It can be seen that the resulting governing equations form a coupled nonlinear system of ordinary differential equations treating $\xi$ as a parameter. These equation are solved numerically using an implicit Runge-Kutta-Butcher [34] initial value solver together with the iteration scheme of Nachtsheim and Swigert [35] as discussed before. Solutions thus obtained for different values of physical parameters, in terms of local skin friction $C_{f} R_{x}^{\frac{1}{2}}$ and the local Nusseltnumber $N u e_{x}^{-\frac{1}{2}}$, which are obtained by the following expressions (38) and (39) respectively, are compared with that obtained in the following section for entire time regime.

$$
u^{\prime \prime}+P_{1} \eta u^{\prime}+P_{2} u^{\prime} v+P_{3}\left(1-u^{2}\right)=P_{4}\left[\frac{\partial u}{\partial \tau}-\lambda G\right]+P_{5}\left[u \frac{\partial u}{\partial \tau}-u^{\prime} \frac{\partial v}{\partial \tau}\right]+P_{6} \xi\left[u \frac{\partial u}{\partial \xi}-u^{\prime} \frac{\partial v}{\partial \xi}\right]+P_{4} M^{2} \xi(1-u)
$$




$$
\frac{1}{P r}+P_{1} \eta G^{\prime}+P_{2} G^{\prime} v-P_{7} G u=P_{4} \frac{\partial G}{\partial \tau}+P_{5}\left[u \frac{\partial G}{\partial \tau}-G^{\prime} \frac{\partial v}{\partial \tau}\right]
$$

And the boundary conditions are:

$$
\begin{gathered}
v(\xi, \tau, 0)=u(\xi, \tau, 0)=0, G(\xi, \tau, 0)=1 \\
u(\xi, \tau, \infty)=1, G(\xi, \tau, \infty)=0
\end{gathered}
$$

Where $P_{1}=e^{-\tau}, P_{2}=(m+1)\left(1-e^{-\tau}\right)+(m-1) \tau e^{-\tau}, P_{3}=2 m\left(1-e^{-\tau}\right), P_{4}=2\left(1-e^{-\tau}\right)$,

$$
P_{5}=2(m-1) \tau\left(1-e^{-\tau}\right), P_{6}=2(1-m)\left(1-e^{-\tau}\right) \text { and } P_{7}=2(2 m-1)\left(1-e^{-\tau}\right)
$$

Now equations (40)-(41) subject to the boundary conditions (42) are discretized using central difference approximation along $\eta$-direction while backward difference is used for both $\xi$-direction and $\tau$-direction. Thus we have a system of tri-diagonal algebraic equations of the form:

$$
A_{k} W_{i, j}^{n}+B_{k} W_{i, j-1}^{n}+C_{k} W_{i, j+1}^{n}=D_{k}(k=1,2)
$$

In the above equations, the subscript $k(=1$ and 2$)$ represents the functions $u$ and $G$ respectively.

Where $A_{k}, B_{k}, C_{k}, D_{k}$ are given as below:

$$
\begin{aligned}
& A_{1}=2+(\Delta \eta)^{2}\left[P_{3} u_{2, j}^{n}+\frac{P_{4}}{\Delta \tau}+\frac{P_{5}}{\Delta \tau} u_{2, j}^{n}+\frac{P_{6}}{\Delta \xi} \xi u_{2, j}^{n}-P_{4} M^{2} \xi\right] \\
& B_{1}=-1+\frac{1}{2} \Delta \eta\left[\frac{P_{1}}{2}[\eta(j)+\eta(j-1)]+P_{2} v_{2, j}^{n}+P_{5} \frac{v_{2, j}^{n}-v_{2, j}^{n-1}}{\Delta \tau}+P_{6} \xi \frac{v_{2, j}^{n}-v_{1, j}^{n}}{\Delta \xi}\right] \\
& C_{1}=-1-\frac{1}{2} \Delta \eta\left[\frac{P_{1}}{2}[\eta(j)+\eta(j-1)]+P_{2} v_{2, j}^{n}+P_{5} \frac{v_{2, j}^{n}-v_{2, j}^{n-1}}{\Delta \tau}+P_{6} \xi \frac{v_{2, j}^{n}-v_{1, j}^{n}}{\Delta \xi}\right] \\
& D_{1}=(\Delta \eta)^{2}\left[P_{3}+\left(P_{4}+P_{5} u_{2, j}^{n}\right) \frac{u_{2, j}^{n-1}}{\Delta \tau}+P_{4} \lambda G_{2, j}^{n}+P_{6} \xi u_{2, j}^{n} \frac{u_{1, j}^{n}}{\Delta \xi}-P_{4} M^{2} \xi\right] \\
& A_{2}=\frac{2}{P r}+\frac{(\Delta \eta)^{2}}{\Delta \tau}\left(P_{4}+P_{5} u_{2, j}^{n}\right) \\
& C_{f} R_{x}^{\frac{1}{2}}=\xi^{\frac{m+1}{2(m-1)}}\left(2\left(1-e^{-\tau}\right)\right)^{-\frac{1}{2}} F^{\prime \prime}(\xi, \tau, 0) \\
& B_{2}=-\frac{1}{P r}+\frac{1}{2} \Delta \eta\left[P_{1}+P_{2} v_{2, j}^{n}+\frac{P_{5}}{\Delta \tau}\left(v_{2, j}^{n}-v_{2, j}^{n-1}\right)\right] \\
& C_{2}=-\frac{1}{P r}-\frac{1}{2} \Delta \eta\left[P_{1}+P_{2} v_{2, j}^{n}+\frac{P_{5}}{\Delta \tau}\left(v_{2, j}^{n}-v_{2, j}^{n-1}\right)\right] \\
& D_{2}=\frac{(\Delta \eta)^{2}}{\Delta \tau} G_{2, j}^{n-1}\left(P_{4}+P_{5} u_{2, j}^{n}\right) \\
& N u \operatorname{Re}_{x}^{-\frac{1}{2}}=-\xi^{\frac{3 m+1}{2(1-m)}}\left(2\left(1-e^{-\tau}\right)\right)^{-\frac{1}{2}} G^{\prime}(\xi, \tau, 0)
\end{aligned}
$$

\begin{tabular}{lllll}
\hline \multirow{2}{*}{$\tau$} & $C_{f} R e_{x}^{1 / 2}$ & $N u R e_{x}^{-1 / 2}$ \\
\cline { 2 - 5 } & All $\boldsymbol{\tau}$ & Small \&Large $\boldsymbol{c}$ & All $\boldsymbol{\tau}$ & Small \&Large $\boldsymbol{\tau}$ \\
\hline & & $\mathrm{M}=0.0$ & & \\
0.01 & 5.58814 & $5.65643 \mathrm{~s}$ & 4.68592 & $4.70704 \mathrm{~s}$ \\
0.10 & 2.05613 & $2.17836 \mathrm{~s}$ & 1.49492 & $1.51200 \mathrm{~s}$ \\
0.50 & 1.48560 & $1.49208 \mathrm{~s}$ & 0.69460 & $0.71662 \mathrm{~s}$ \\
1.00 & 1.51896 & $1.54361 \mathrm{~s}$ & 0.52669 & $0.55475 \mathrm{~s}$ \\
2.00 & 1.56499 & $1.59030 \mathrm{a}$ & 0.47531 & $0.49731 \mathrm{a}$ \\
3.00 & 1.56498 & $1.58924 \mathrm{a}$ & 0.47743 & $0.48489 \mathrm{a}$ \\
4.00 & 1.56393 & $1.58924 \mathrm{a}$ & 0.47900 & $0.48151 \mathrm{a}$ \\
6.00 & 1.56433 & $1.57816 \mathrm{a}$ & 0.47870 & $0.48005 \mathrm{a}$ \\
\hline
\end{tabular}




\begin{tabular}{lllll}
\hline \multirow{3}{\tau}{$\tau$} & $C_{f} R e_{x}^{1 / 2}$ & \multicolumn{3}{c}{$N u R e_{x}^{-1 / 2}$} \\
\cline { 2 - 5 } & All $\boldsymbol{\tau}$ & Small \&Large $\boldsymbol{\tau}$ & All $\boldsymbol{~}$ & Small \&Large $\boldsymbol{\tau}$ \\
\hline 8.00 & 1.56474 & $1.57688 \mathrm{a}$ & 0.47820 & $0.48003 \mathrm{a}$ \\
10.00 & 1.56472 & $1.57641 \mathrm{a}$ & 0.47815 & $0.48025 \mathrm{a}$ \\
& & $\mathrm{M}=1.0$ & & \\
0.01 & 5.52785 & $5.65821 \mathrm{~s}$ & 4.68377 & $4.72704 \mathrm{~s}$ \\
0.10 & 1.88133 & $1.95520 \mathrm{~s}$ & 1.49429 & $1.53200 \mathrm{~s}$ \\
0.50 & 1.15290 & $1.39661 \mathrm{~s}$ & 0.69480 & $0.71662 \mathrm{~s}$ \\
1.00 & 1.15048 & $1.23353 \mathrm{~s}$ & 0.52625 & $0.58831 \mathrm{~s}$ \\
2.00 & 1.19360 & $1.15941 \mathrm{a}$ & 0.46625 & $0.52276 \mathrm{a}$ \\
3.00 & 1.18908 & $1.14670 \mathrm{a}$ & 0.46372 & $0.49398 \mathrm{a}$ \\
4.00 & 1.18646 & $1.14250 \mathrm{a}$ & 0.46393 & $0.49196 \mathrm{a}$ \\
6.00 & 1.18674 & $1.13975 \mathrm{a}$ & 0.46344 & $0.49153 \mathrm{a}$ \\
8.00 & 1.18717 & $1.13893 \mathrm{a}$ & 0.46303 & $0.49191 \mathrm{a}$ \\
10.00 & 1.18701 & $1.13860 \mathrm{a}$ & 0.46302 & $0.49233 \mathrm{a}$ \\
\hline
\end{tabular}

\section{Results and Discussion}

In this present study, the mixed convection flows of an electrically conducting, viscous and incompressible fluid past a wedge in presence of magnetic field have been analyzed. The governing equations have been solved by using the straight forward finite difference method for the entire time regime. Numerical solutions are also obtained by using the perturbation method for small time regime and the asymptotic method for large time regime. The obtained numerical results are presented with the help of graphical illustrations as well as in tabular form.

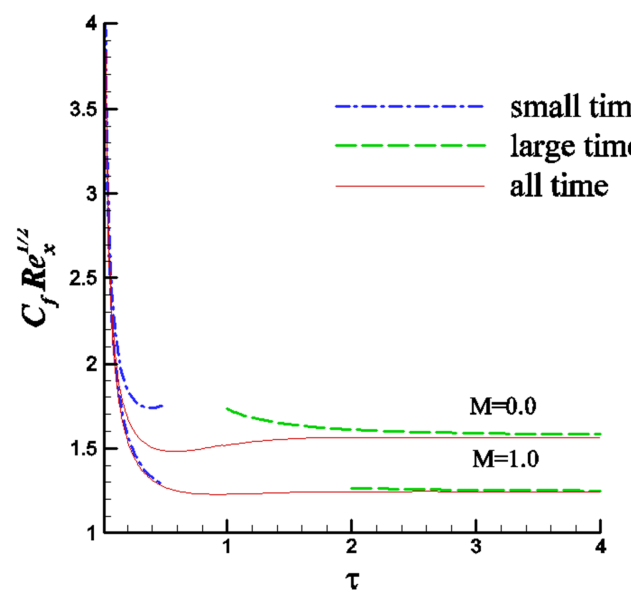

(a)

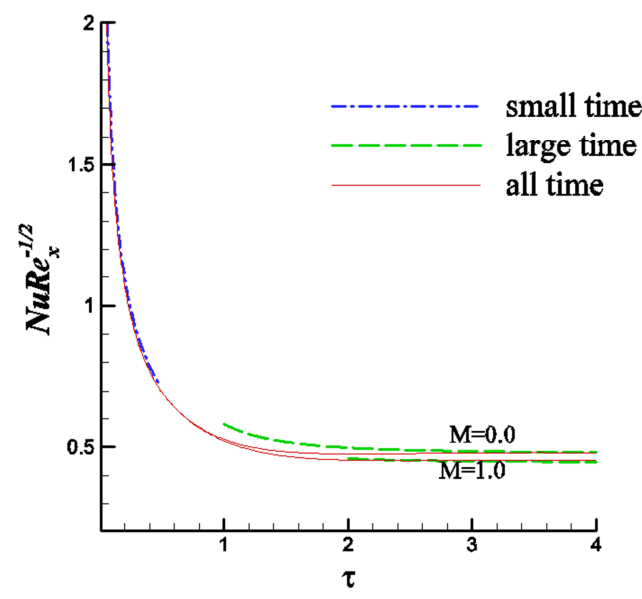

(b)

Figure 2. (a) Local skin friction and (b) Local Nusselt number for different values of magnetic parameter Magainst $\tau$ forPr $=0.72, m=0.5$ and $\lambda=0.5$ at $\xi=0.3$ obtained by three distinct methods.

With the purpose of ensuring the numerical solution, a comparison of the (a) local skin friction and (b) local Nusselt number obtained by the stream function formulation and the series solutions for small and large $\tau$ is shown in Figure 2, Figure 3 as well as in Table 1. It is evident from the figures that the solutions are in excellent agreement.

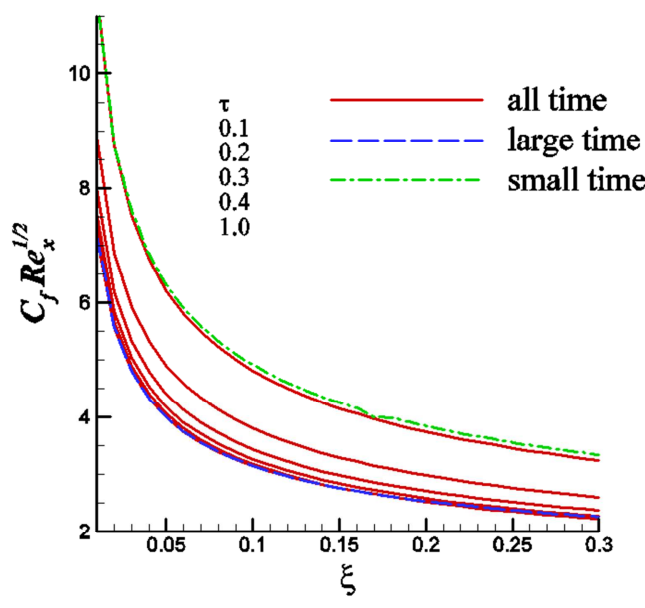

(a)

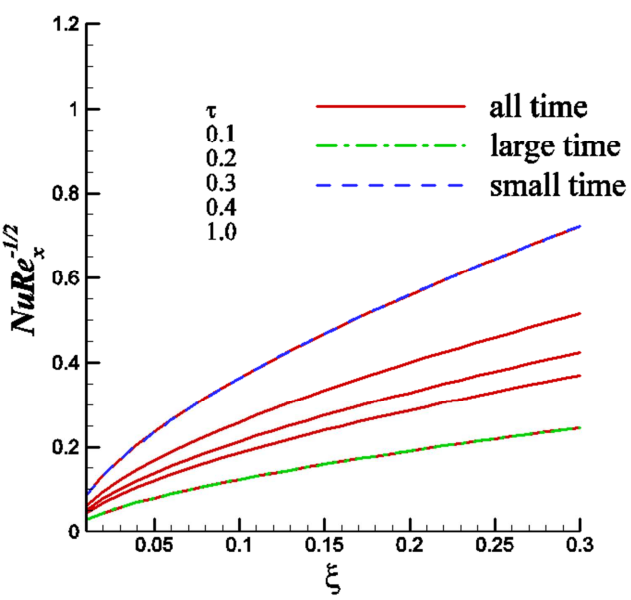

(b)

Figure 3. (a) Local skin friction and (b) Local Nusselt number against $\xi$ at different time steps for $P r=0.72, M=1.0, m=0.5$ and $\lambda=0.5$ obtained by three distinct methods.

Figure 2(a) and 2(b) demonstrate the effects of magnetic field on the local skin friction coefficient, $C_{f} R e_{x}^{1 / 2}$, and the local Nusselt number, $N u R e_{x}^{-1 / 2}$, against $\tau$ respectively while $\operatorname{Pr}=0.7, \xi=0.3, m=0.5$ and $\lambda=0.5$. From these figures it is observed that when magnetic parameter increases, then both 
the local skin-friction and local Nusselt number decrease.

The effects of parameter $\xi$ on the local skin friction coefficient, $C_{f} R e_{x}^{1 / 2}$, and the local Nusselt number, $N u R e_{x}^{-1 / 2}$, from the surface at different time steps are shown in Figure 3(a) and 3(b) respectively. In this figure the physical

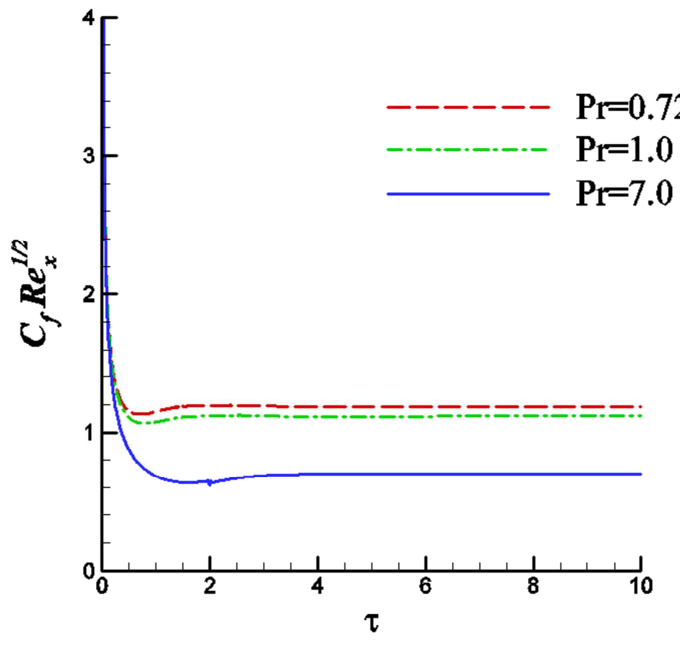

(a) parameters are chosen to be $P r=0.72, M=1.0, m=0.5$ and $\lambda$ $=0.5$. It is observed from this figure that the local skin friction is decreased considerably while the local Nusselt number increases as $\xi$ increases.

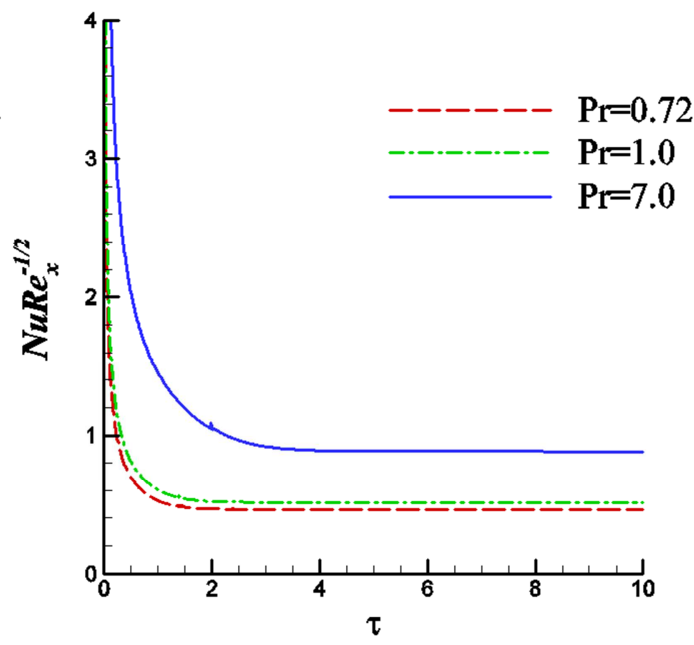

(b)

Figure 4. (a) Local skin friction and (b) Local Nusselt number against $\tau$ for different values of Prandtl number Pr while M=1.0, $m=0.5$ and $\lambda=0.5$ at $\xi=0.3$.

Figure 4(a) and 4(b) exhibits the effects of varying the Prandtl number, $\operatorname{Pr}(=0.72,1.0,7.0)$, on the local skin friction coefficient, $C_{f} R e_{x}^{1 / 2}$, and the local Nusselt number, $N u R e_{x}^{-1 / 2}$, against $\tau$ respectively at $M=1.0, m=0.5, \lambda=0.5$ and $\xi=0.3$. Figure 4(a) shows that an increase in the value of

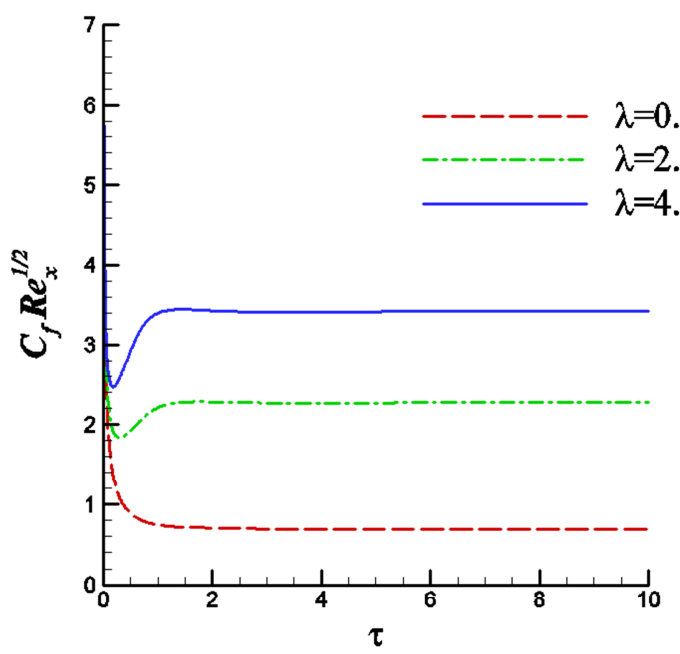

(a)
Prandtl number, $P r$, leads to a decrease in the value of local skin friction coefficient. And from Figure 4(b) it can be observed that the value of local Nusselt number coefficient increases with the increasing values of Prandtl number.

Figure 5.(a) Local skin friction and (b) Local Nusselt number against $\tau$ for different values of $\lambda$ while $M=1.0, m=0.5$ and $\operatorname{Pr}=0.72$ at $\xi=0.3$.

Figure 5(a) and 5(b) illustrate the effects of changing the Richardson number, $\lambda(=0.0,2.0,4.0)$, on the local skin friction coefficient, $C_{f} R e_{x}^{1 / 2}$, and the local Nusselt number, $N u R e_{x}^{-1 / 2}$, against $\tau$ respectively at $M=1.0, m=0.5, P r=0.72$ and $\xi=0.3$. Figure 5(a) and 5(b) show that an increase in the value of Richardson number, $\lambda$, leads to an increase in thevalue of local skin friction coefficient and the value of local Nusselt number coefficient respectively. 


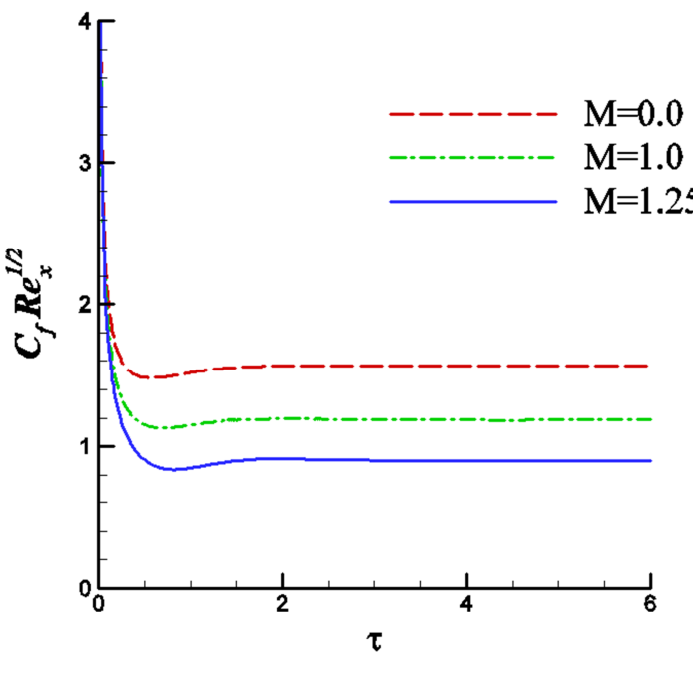

(a)

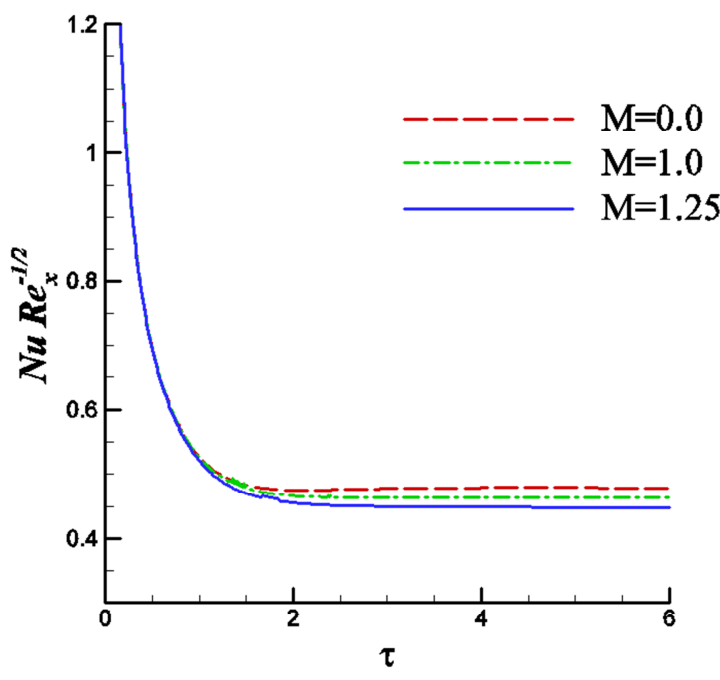

(b)

Figure 6. (a) Local skin friction and (b) Local Nusselt number for different values of magnetic parameter Magainst $\tau$ forPr $=0.72, m=0.5$ and $\lambda=0.5$ at $\xi=0.3$.

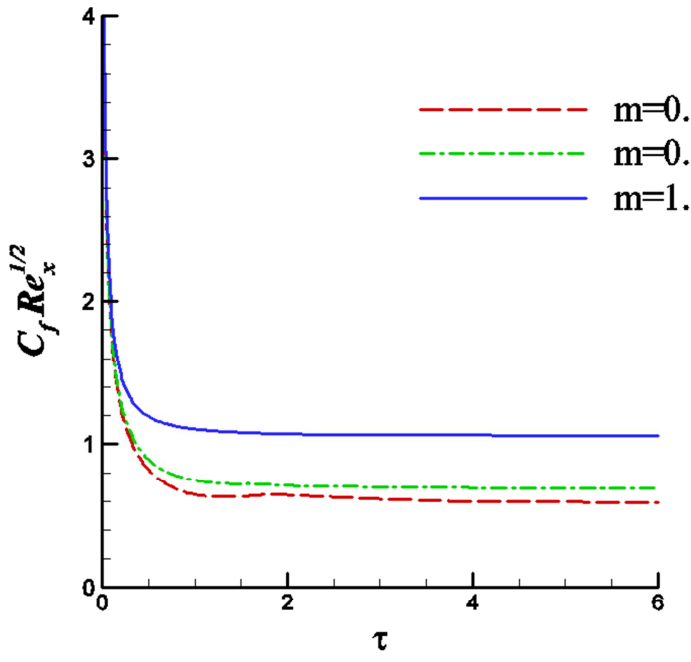

(a)

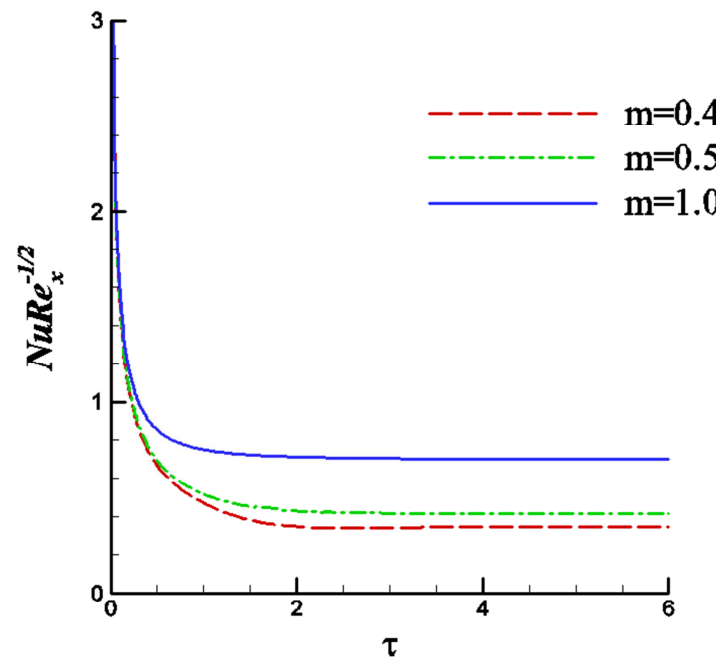

(b)

Figure 7. (a) Local skin friction and (b) Local Nusselt number for different values of exponent $m$ against $\tau$ for $\operatorname{Pr}=0.72, M=1.0$ and $\lambda=0.5$ at $\xi=0.3$.

Figure 6(a) and 6(b), we can see that when magnetic parameter increases, both the local skin The effects of varying values of the magnetic parameter, $M(=0.0,1.0,1.25)$ on the local skin friction coefficient, $C_{f} R e_{x}^{1 / 2}$, and the local Nusselt number, $N u R e_{x}^{-1 / 2}$, against $\tau$ are displayed in Figure 6(a) and 6(b) for the case of $\operatorname{Pr}=0.72, m=0.5$ and $\lambda=0.5$ at $\xi=0.3$. By observing the friction coefficient and the local Nusselt number coefficient decreases

The effects of varying values of the exponent, $m(=0.4,0.5$, 1.0) on the local skin friction coefficient, $C_{f} R e_{x}^{1 / 2}$, and the local Nusselt number, $N u R e_{x}^{-1 / 2}$, against $\tau$ are displayed in Figure 7(a) and 7(b) for the case of $P r=0.72, M=1.0$ and $\lambda$ $=0.5$ at $\xi=0.3$. Figure 7(a) and 7(b) show that an increase in the value of $m$, results in increasing the value of local skin friction coefficient and the value of local Nusselt number coefficient respectively.

\section{Conclusion}

In this study it has been sought to determine how the presence of magnetic parameter affects the mixed convection unsteady two-dimensional boundary layer flow and heat transfer past a wedge with variable surface temperature. Solutions of the governing local nonsimilarity equations are obtained by three distinct methodologies, namely the perturbation method for small time $\tau$, the asymptotic solution method for large time $\tau$ and the finite difference method of all time $\tau$. Detailed numerical calculations have been carried out and presented in terms of local skin friction and Nusselt number. In general it is seen that the asymptotic solutions for small and large values of $\tau$ are in excellent agreement with the finite difference solutions.

From the above investigations, the following conclusions may be drawn: 
1. An increase in the value of $M$ serves to decrease both the local skin friction and local Nusselt number.

2. An increase in the value of mleads to increase both the local skin friction and local Nusselt number.

3. An increase in the value of Richardson number, $\lambda$, leads to an increase in the value of local skin friction coefficient as well as the value of local Nusselt number coefficient.

4. An increase in the value of Prandtl number, $P r$, leads to a decrease in the value of local skin friction coefficient and the value of local Nusselt number coefficient increases with the increasing values of Prandtl number.

\section{References}

[1] L. G. Leal, Laminar Flow and Convective Transport Phenomena: Scaling Principles and Asymptotic Analysis, Butterworth Heinemann, Boston, (1992).

[2] K. Gersten, H. Herwig, V. Stramungsmechanik, Braunschweig, Wiesbaden (1992).

[3] H. Schlichting, K. Gersten, Grenzschicht-Theorie, Springer-Verlag, Berlin, (1997).

[4] V. M. Falkner and S. W. Skan, Some Approximate Solutions of the Boundary-Layer Equations, Philosophical Magazine, 12 (1930) 865-896.

[5] D. R. Hartree, On an equation occurring in Falkner and Skan's approximate treatment of the equations of the boundary layer. In Mathematical Proceedings of the Cambridge Philosophical Society, Cambridge University Press, 33(1937) 223-239.

[6] J. C. Y. Koh and J. P. Hartnett, Skin friction and heat transfer for incompressible laminar flow over porous wedges with suction and variable wall temperature, International Journal of Heat and Mass Transfer 2 (1961) 185-198.

[7] N. G. Kafoussias and N. D. Nanousis, Magnetohydrodynamic laminar boundary-layer flow over a wedge with suction or injection, Canadian Journal of Physics 75.10 (1997) 733-745.

[8] M. Kumariand Rama Subba Reddy Gorla, Combined convection along a non-isothermal wedge in a porous medium, Heat and Mass Transfer 32.5 (1997) 393-398.

[9] M. A. Hossain, MdSazzadMunir, and David Andrew S. Rees, Flow of viscous incompressible fluid with temperature dependent viscosity and thermal conductivity past a permeable wedge with uniform surface heat flux, International journal of thermal sciences 39.6 (2000) 635-644.

[10] Mahesh Kumari, Harmindar S. Takhar, and GirishwarNath, Mixed convection flow over a vertical wedge embedded in a highly porous medium, Heat and Mass Transfer 37.2 (2001) 139-146.

[11] N. Riley, Unsteady viscous flows. Science Progress 74 (1990) 361-377.

[12] D. R. Telionis, Unsteady viscous flows, Springer-Verlag, New York, 1997.

[13] David K. Ludlow, Peter A. Clarkson, and Andrew P. Bassom, New similarity solutions of the unsteady incompressible boundary-layer equations, Quarterly Journal of Mechanics and Applied Mathematics 53.2 (2000) 175-206.
[14] Stuart H Smith, The impulsive motion of a wedge in a viscous fluid, ZeitschriftfürAngewandteMathematik und Physik (ZAMP) 18.4 (1967) 508-522.

[15] Kenichi Nanbu, Unsteady Falkner-Skan flow, ZeitschriftfürangewandteMathematik und Physik ZAMP 22.6 (1971) 1167-1172.

[16] M. A. Hossain, Bhowmick S, Gorla RS. Unsteady mixed-convection boundary layer flow along a symmetric wedge with variable surface temperature. International journal of engineering science44(10) (2006) 607-620.

[17] M. G. Hall, The boundary layer over an impulsively started flat plate, Proceedings of the Royal Society of London A: Mathematical, Physical and Engineering Sciences 310(1969) 401-414.

[18] Simon D. Harris, Derek B. Ingham, and Ioan Pop, Unsteady heat transfer in impulsive Falkner-Skan flows: constant wall temperature case, European Journal of Mechanics-B/Fluids 21.4 (2002) 447-468.

[19] H. B. Keller and T. Cebeci, Accurate Numerical Methods for Boundary Layer Flows, I. Two-Dimensional Laminar Flows, Lecture Notes in Physics, Proceedings of 2nd International Conference on Numerical Methods in Fluid Dynamics, Springer-Verlag, Berlin. (1971).

[20] E. M. Sparrow and R. D. Cess, The effect of a magnetic field on free convection heat transfer, International Journal of Heat and Mass Transfer 3.4 (1961) 267-274.

[21] K. R. Singh and T. G. Cowling, Thermal convection in magnetohydrodynamic bounadary layers, J. Mech. Appl. Math. 16(1963) 1-5.

[22] GrahamWilks, Magnetohydrodynamic free convection about a semi-infinite vertical plate in a strong cross field, Zeitschriftfür angewandte Mathematik and Physik, ZAMP 27.5 (1976) 621-631.

[23] M. H. Cobble, Free convection with mass transfer under the influence of a magnetic field, Nonlinear Analysis: Theory, Methods \& Applications 3.1 (1979) 135-143.

[24] M. A. Hossain, K. C. A. Alam, and D. A. S. Rees, MHD forced and free convection boundary layer flow along a vertical porous plate, Applied Mechanics and Engineering 2.1 (1997) 33-51.

[25] P. Ganesanand G. Palani, Finite difference analysis of unsteady natural convection MHD flow past an inclined plate with variable surface heat and mass flux, International journal of heat and mass transfer 47.19 (2004) 4449-4457.

[26] G. Palani and Kwang-Yong Kim, The effects of MHD on free-convection flow past a semi-infinite isothermal inclined plate, Journal of Engineering Physics and Thermophysics 81.4 (2008) 724-731.

[27] S. M. Mahfooz, M. A. Hossain, and Rama Subba Reddy Gorla, Radiation effects on transient magnetohydrodynamic natural convection flow with heat generation, International Journal of Thermal Sciences 58 (2012) 79-91.

[28] L. K. Saha, M. A. Hossain, and Rama Subba Reddy Gorla, Effect of Hall current on the MHD laminar natural convection flow from a vertical permeable flat plate with uniform surface temperature, International journal of thermal sciences 46.8 (2007) 790-801. 
[29] SP Anjali Devi and R. Kandasamy, Thermal stratification effects on laminar boundary-layer flow over a wedge with suction or injection, Mechanics Research Communications 28.3 (2001) 349-354.

[30] H. Schlichting, K. Gersten, E. Krause and H. Oertel, Boundary-layer theory, New York(1960).

[31] Nepal C. Roy and M. Anwar Hossain, Unsteady Magnetohydrodynamic Mixed Convection Flow of Micropolar Fluid Past a Permeable Sphere, Journal of Thermophysics and Heat Transfer (2017).

[32] Kai-Long Hsiao, MHD mixed convection for viscoelastic fluid past a porous wedge, International Journal of Non-Linear Mechanics 46.1 (2011) 1-8.

[33] S. Siddiqaand M. A. Hossain, Mixed convection boundary layer flow over a vertical flat plate with radiative heat transfer, (2012).

[34] John C. Butcher, Implicit Runge-Kutta processes, Mathematics of Computation, 18.85 (1964) 50-64.

[35] Philip R. Nachtsheim and Paul Swigert, Statisfaction of asymptotic boundary conditions in numerical solution of systems of nonlinear equations of boundary-layer type, NASA TND-3004(1965). 